\title{
Segmentation/reconstruction of range images based on piecewise-linear approximation
}

\author{
Enrico Puppo \\ Istituto per la Matematica Applicata - Consiglio Nazionale delle Ricerche \\ Via De Marini, 6 (Torre di Francia) - 16149 Genova - ITALY \\ Email: puppo@ima.ge.cnr.it
}

\begin{abstract}
A technique for segmenting and reconstructing range images of polyhedral objects is presented. A piecewise-linear approximation based on a triangulation of the image is computed first. Optimization techniques are applied next to detect jump and crease edges, to eliminate false boundaries, and to merge nearly coplanar triangles into larger polygonal patches.
\end{abstract}

\section{Introduction}

The visible portion of a real world scene framed by a range image is a piecewisesmooth surface defined by the graph of a bivariate function, with discontinuities of various order along characteristic lines. The range image is a noisy, sampled version of the surface. In order to extract useful information, the image plane can be segmented into coherent regions, corresponding to homogeneous smooth surfaces in the scene, and each surface patch can be reconstructed through a suitable function that fits data.

Several techniques for segmenting and reconstructing range images have been developed in the literature (e.g., $[1,3,4,6])$. Here, we propose a surface-oriented method that combines refinement and aggregation to provide a segmentation of a range image, and its representation through a piecewise-linear surface.

An important characteristic of this method is the use of irregular triangulations to segment the image. Triangulations result more adaptive than recursive decomposition schemes based on regular shapes (e.g., quadtrees), which are traditionally used in split-and-merge methods. Triangulations have been used extensively to reconstruct continuous surfaces, but they have been seldom applied to image processing. A recent triangulation-based reconstruction technique, which allows to deal with surface discontinuities has been proposed in [3]. Such a technique uses traditional contour-oriented segmentation and labeling techniques to detect characteristic lines in the image, which are used next to compute a constrained triangulation: much work is performed at pixel level, while limiting the use of triangulations to a final reconstruction stage. A peculiarity of the method proposed here is that image pixels are considered only during an initial refinement stage to build a fragmentation of the image into triangles. The rest of processing performs aggregation on a subdivision of the image into polygonal regions (initially trianlges), while disregarding the underlying pixel grid. 


\section{Problem definition}

An orthographic range signal of a scene is a piecewise-smooth function $f: \mathbb{R}^{2} \longrightarrow$ $\mathbb{R}$ defined as follows: for each point $X$ in a frame (i.e., a rectangular window on a plane) $D \subset \mathbb{R}^{2}, f(X)$ is the distance between $X$ and the first point of an object in the framed scene that is hit by a ray through $X$ and perpendicular to the frame plane. Assuming that the framed scene is composed of objects bounded by piecewise-smooth surfaces, $f$ is describable by a partition of $D$ into a finite number of regions $\mathcal{R}=\left\{R_{1}, \ldots, R_{k}\right\}$, such that $f$ is represented over each region $R_{i}$ by a smooth function $\phi_{i}$ belonging to family $\mathcal{F}=\left\{\phi_{1}, \ldots, \phi_{k}\right\}$. The pair $(\mathcal{R}, \mathcal{F})$ is called a representation of $f . \mathcal{R}$ is called a segmentation of $f$ if and only if it is composed of a minimum number of maximal regions, otherwise it is called a fragmentation of $f$. Note that $f$ can have discontinuities of various order along the boundaries of regions of $\mathcal{R}$ : a discontinuity of the 0th order is called a jump, while a discontinuity of the 1st order is called a crease.

Given a zero-mean random noise process $n$, we call $\tilde{f}=f+n$ a noisy signal of $f$, and we consider a range image as a sampled version of $\tilde{f}$. A regular grid over a frame $D$ is a subdivision of $D$ into rectangular regions of uniform size, called pixels; the points at the corners of pixels are called vertices. A range image $I$ of $\tilde{f}$ is a restriction of $\tilde{f}$ to the centers of pixels, and it is represented as a two dimensional array of $N \times N$ values, where $I_{i j}=\tilde{f}(x(i), y(j))$, and the vector function $(x(), y())$ gives the centers of all pixels for $i, j \in\{1, \ldots, N\}$.

Let $\hat{f}$ be a real-valued function described on $D$ by $(\hat{\mathcal{R}}, \hat{\mathcal{F}})$. The error in approximating $\tilde{f}$ by $\hat{f}$ on the basis of $I$ is defined as

$$
E(\hat{f})=\max _{i, j=1 \ldots N}\left|\hat{f}(x(i), y(j))-I_{i j}\right| .
$$

The approximate segmentation/reconstruction problem for $I$, at precision $\varepsilon>0$ is to find a minimal representation $(\hat{\mathcal{R}}, \hat{\mathcal{F}})$, such that $E(\hat{f})<\varepsilon$.

Ideally, all discontinuities of $f$ - and only such lines - should appear as boundaries of regions of $\hat{\mathcal{R}}$. In practice, the precision threshold will influence the quality of the result. The value of $\varepsilon$ can be set according to the expected variance of the noise affecting $I$ : in general, the smaller its value, the larger the number of regions in $\mathcal{T}$, and the smaller their size.

In the following, polyhedral scenes, i.e., signals that can be represented in terms of polygonal subdivisions and linear functions, will be considered. The method we propose is also useful ato obtain approximate polygonal segmentatations, and piecewise-linear reconstructions of scenes with curved objects.

\section{Adaptive image triangulation}

The first stage of the method (refinement phase) consists of an algorithm to compute a fragmentation $\mathcal{T}$ of $I$ into triangular regions having their vertices at vertices of the pixel grid. $\mathcal{T}$ is called a triangulation of $I$. In order to be effective, $\mathcal{T}$ should be formed by a restricted number of triangles, whose edges form chains that approximate all edges of the desired segmentation $\mathcal{R}$. 
Given $\mathcal{T}$, a piecewise-linear fragmented surface $f_{\mathcal{T}}$ is defined as follows. For each triangle $t$ of $\mathcal{T}$, for each vertex $v_{i}$ of $t, i=0,1,2$, consider the pixel $X_{i}$ incident into $v_{i}$ and whose intersection with $t$ has maximum area. $f_{\mathcal{T}}$ is defined over $t$ as the linear function interpolating range values at the centers of pixels $X_{i}, i=0,1,2$, restricted to $t$. Note that different triangles incident into a vertex $v$ can have different range values at $v$, thus yielding a possible jump at every edge of $\mathcal{T}$ (see Figure 1a).

We are interested in finding $\mathcal{T}$ such that $E\left(f_{\mathcal{T}}\right) \leq \varepsilon$ for a given $\varepsilon \geq 0 . \mathcal{T}$ is built based on a simple heuristic technique that iteratively refines an initial triangulation covering $D$ by inserting one new vertex at a time, until the required precision is reached. This algorithm is reminiscent of a technique proposed first in [5] to reconstruct continuous surfaces.

This refinement technique naturally tends to select points along characteristic lines of the image, hence approximating such lines through edges of the triangulation. Such good behavior, which had already been demonstrated in the literature for continuous surfaces, is confirmed in our experiments for the noncontinuous case of range data.

\section{Elimination of false boundaries}

Although characteristic lines of $I$ are approximated by chains of edges of $\mathcal{T}$, many other edges of $\mathcal{T}$ do not correspond to line features of $I$, and many false jumps are present in the reconstructed surface. False jumps are eliminated through local analysis of range values of the reconstructed surface at the vertices of $\mathcal{T}$.

Let $t_{i_{1}}, \ldots, t_{i_{h}}$ be the set of triangles incident into a vertex $v_{i}$ of $\mathcal{T}$, in counterclockwise order around $v_{i}$, and let $\phi_{i_{1}}, \ldots, \phi_{i_{h}}$ be the linear restrictions of $f_{\mathcal{T}}$ to such triangles. The values $P_{i_{j}}=\phi_{i_{j}}\left(v_{i}\right), j=1, \ldots, h$, are in general all different (see Figure 2a): if $P_{i_{j}} \neq P_{i_{j+1}}$ then $f_{\mathcal{T}}$ is necessarily discontinuous along the edge common to $t_{i_{j}}$ and $t_{i_{j+1}}$. We wish to maintain such a discontinuity only if it corresponds to a jump. In order to eliminate a discontinuity, we need to make $P_{i_{j}}$ and $P_{i_{j+1}}$ equal. We consider the sequence $P_{i_{0}}, P_{i_{1}}, \ldots, P_{i_{h}}$, where $P_{i_{0}}=P_{i_{h}}$, as a noisy sample of a piecewise-constant univariate signal at points $0,1, \ldots, h$ : each constant segment of the signal spans all $P_{i_{j}}$ s that should share the same range value. We reconstruct the signal in the interval $[0, h]$ through a function $u_{i}$ that fits data, and that satisfies the constraint $u_{i}(0)=u_{i}(h)$.

The technique used to reconstruct $u_{i}$ is a modification of the weak elastic string described in [2]: $u_{i}$ is modeled as an elastic string under weak continuity constraints; breaks are placed in the string where the continuity constraint is violated. An energy is associated to the string, which is composed of three terms:

$P:$ the sum of penalties $\alpha$ for each discontinuity $P=\sum_{j=1}^{h} \alpha l_{i_{j}}$, where $l_{i_{j}}=1$ if there is a discontinuity between $P_{i_{j-1}}$ and $P_{i_{j}}$, and $l_{i_{j}}=0$ otherwise.

$D$ : a weighted measure of faithfulness to data $D=\sum_{j=0}^{h} w_{i_{j}}\left(u_{i}(j)-P_{i_{j}}\right)^{2}$, where $w_{i_{j}}$ is a weight proportional to the area of triangle $t_{i_{j}}$.

$S$ : a measure of slant $S=\lambda^{2} \sum_{j=1}^{h}\left(u_{i}(j)-u_{i}(j-1)\right)^{2}\left(1-l_{i_{j}}\right)$. 


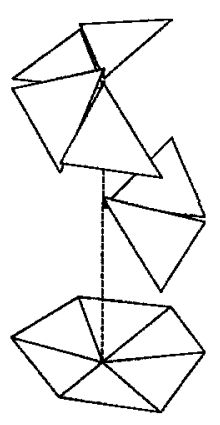

(a)

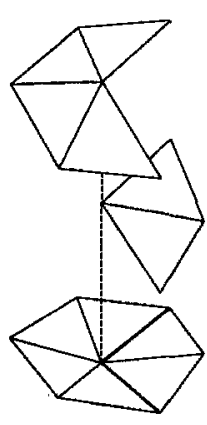

(b)

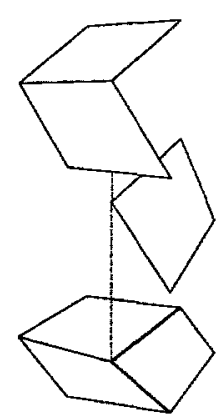

(c)

Fig. 1. Patches incident into a vertex (a) before optimization, (b) after jump detection: thick edges are classified as jumps, (c) after crease detection: dummy edges have been eliminated.

Parameter $\alpha$ is a coefficient of strength of the string: the higher ts value, the less discontinuities we get. Weights $w_{i}$ tends to maintain the result faithful to range values of large triangular patches. Parameter $\lambda$ is aimed to prevent continuous segments of $u_{i}$ to be slanted.

The non-convex energy of the string $E=D+S+P$ must be minimized. To this aim, we have used an iterative algorithm that implements the Graduated Non-Convexity technique proposed in [2], whose details are omitted here for brevity.

Jump edges are detected by applying such operation to all vertices of the triangulation (see Figure $2 \mathrm{~b}$ ). Remaining edges of $\mathcal{T}$ must be classified next into crease edges, and dummy edges, i.e. edges separating nearly coplanar patches.

Detection of creases is performed through a similar optimization technique, although this time an energy is computed over the whole surface. We consider the piecewise linear function $f_{\mathcal{T}}$ currently defined on the triangulation, and we fit to such function another piecewise-linear function $\hat{f}$ that minimizes an energy depending on its distance from $f_{\mathcal{T}}$, on its "smoothness", and on the amount of creases in it. Function $\hat{f}$ is obtained by modifying the range values assumed by $f_{\mathcal{T}}$ at the vertices of triangles, while maintaining the continuity along non-jump edges. Details are omitted here for brevity.

After identifying creases, patches adjacent along dummy edges are merged. The resulting structure is a planar subdivision of the image frame, made of polygonal regions (see Figure 1c).

Each tiny region, whose area is smaller than a given threshold, is merged next into one of its adjacent region, based on maximum likelihood of surfaces. Finally, contours of regions in the subdivision are simplified through a simple line simplification algorithm. 


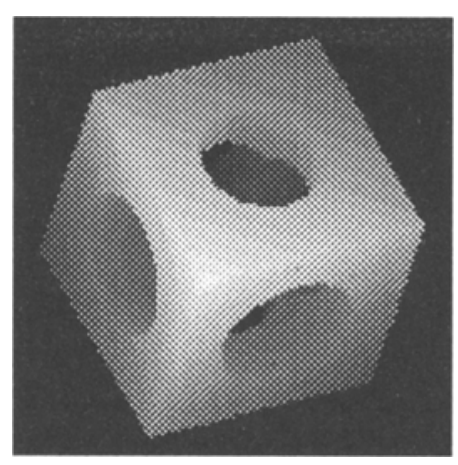

Fig. 2. The input image image rendered as a grey level map.

\section{Experimental results}

The algorithm proposed has been tested on several range images. We show results obtained on a popular test image representing a frame of a drilled cube, containing both plane and curved surfaces (see Figure 2). The image is composed of $128 \times 128$ integer values in the range $[0,255]$.

The fragmentation of the image obtained after the triangulation stage with $\varepsilon=3.84$ (i.e., $1.5 \%$ of the input range) has 971 vertices, i.e., nearly $6 \%$ of the input grid. Most edges at this stage appear as jumps. The main lineal features of the image are approximated by chains of edges: edges of the cube and borders of the holes are clearly visible. After detection of jumps and creases, and deletion of dummy edges, all important features have been retained, although some of them are approximated by jagged chains; some spurious creases have been detected. Figure 3 shows the different stages of reconstruction.

\section{Concluding remarks}

The results presented are relative to an initial study, which is limited to piecewiselinear surfaces, and thus it is suitable to handle polyhedral scenes. Approximate reconstructions of scenes containing objects with curved surfaces can be obtained as well. An immediate extension consists in using higher order polynomial patches over triangles. Curved patches permit to handle a broader class of range images containing curved surfaces.

The method proposed is promising, though we believe that its performances can be highly improved, both in the quality of the results, and in terms of running time.

A further possibility is to adapt a hierarchical triangulation methods [7] to non-continuous surfaces, in order to obtain a multi-scale pyramidal model based on layers of triangulations at different levels of resolution. 

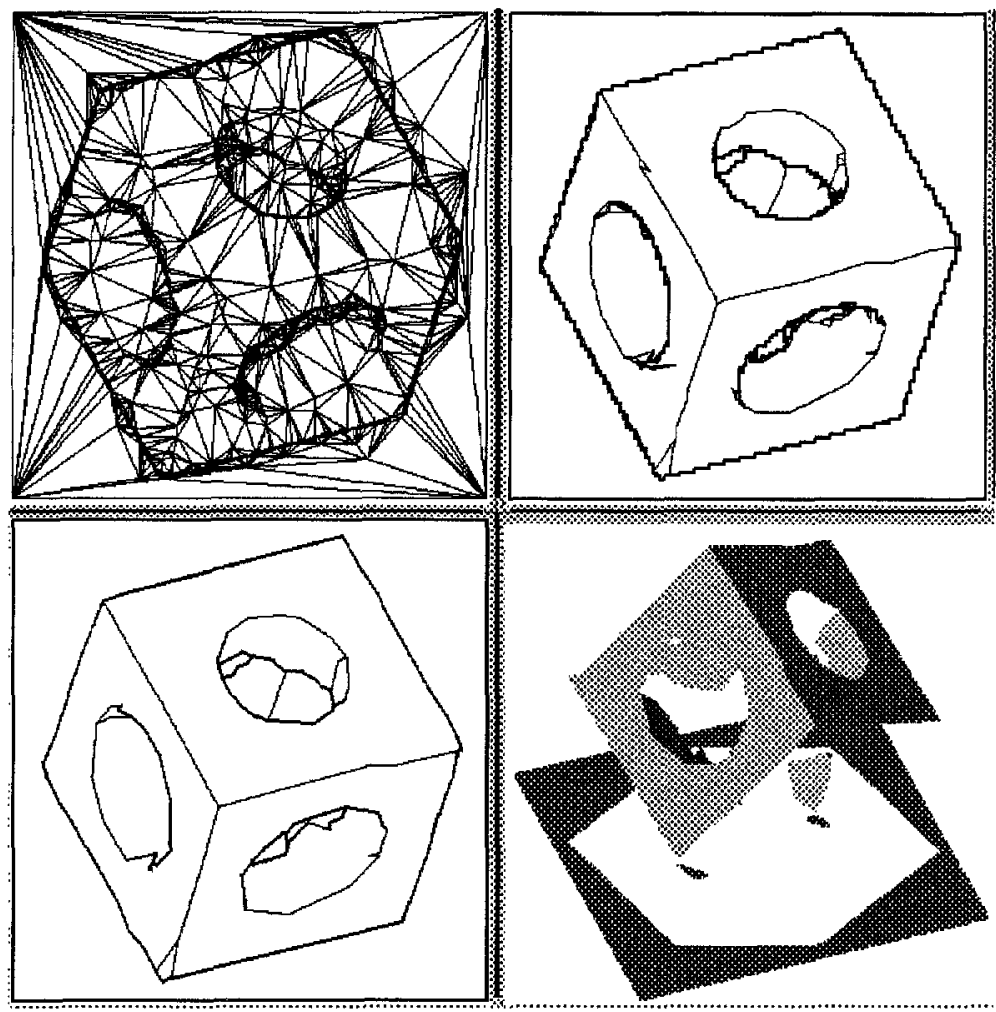

Fig. 3. Different stages of the segmentation: (a) fragmentation after triangulation; (b) segmentation after the detection of jumps and creases (jumps are depicted as bold lines; dummy edges have been removed); (c) final segmentation; (d) a view of the final reconstruction.

\section{References}

1. Besl, P.J., Jain, R.C., Segmentation Through Variable-Order Surface Fitting, IEEE-PAMI, 10, 2 (1988) 167-192.

2. Blake, A., Zisserman, A., Visual Reconstruction, The MIT Press, Cambridge, MA (1987).

3. Chen, X., Schmitt, F., Adaptive range data approximation by constrained surface triangulation, in Modeling in Computer Graphics, B. Falcidieno, T.L. Kunii (Eds.), Spriger-Verlag, Berlin Heidelberg (1993) pp.95-114.

4. Fan, T., Medioni, G., Nevatia, R., Recognizing 3-D Objects Using Surface Descriptions, IEEE-PAMI, 11, 11 (1989) pp.1140-1157.

5. Fowler, R.J., Little, J.J., Automatic extraction of irregular network digital terrain models, ACM Computer Graphics, 13, 3 (1979) pp.199-207.

6. Hoffman, R., Jain, A.K., Segmentation and Classification of Range Images, IEEEPAMI, 9, 5 (1987) pp.608-620.

7. Scarlatos, L., Pavlidis, T., Hierarchical triangulation using cartographic coherence, CVGIP: Graphical Models and Image Processing, 54, 2 (1992) pp.147-161. 